\title{
Análisis Psicométrico de una Escala de Ciberacoso para Población Mexicana
}

\author{
Psychometric Analysis of a Cyberbullying Scale for the Mexican Population
}

\author{
Francisco Quiñonez-Tapia ${ }^{1}$ y María de Lourdes Vargas-Garduño ${ }^{2}$
}

\begin{abstract}
Resumen
El objetivo fue analizar las propiedades psicométricas de las puntuaciones de una escala de ciberacoso aplicada en población mexicana. La muestra fue de 33289 participantes. Se analizaron los indicadores de confiabilidad con el Coeficiente Omega y la validez con el Análisis Factorial Confirmatorio a partir del estimador WLSMV. Se revisó la invarianza para distintos grupos con el Análisis Factorial Confirmatorio Multigrupo, obteniendo un Omega de .948. Los estudios realizados permitieron evidenciar su confiabilidad y validez con una estructura factorial de un solo factor. El análisis multigrupo indicó invarianza considerando variables de: sexo, estadios del desarrollo, escolaridad y lugar de residencia. Los análisis de las puntuaciones de la Escala de Ciberacoso arrojaron evidencias de que es confiable, válida e invariante en la población mexicana. La escala permitirá contar con medidas confiables del ciberacoso para realizar estudios que determinen relaciones causa-efecto con otras variables, así como la prevalencia.
\end{abstract}

Palabras clave: tecnologías de la información y la comunicación, ansiedad, depresión, víctima, violencia

\begin{abstract}
The objective was to analyze the psychometric properties of the scores on a cyberbullying scale applied to the Mexican population. The sample was made up of 33,289 participants. The reliability indicators were analyzed with the Omega Coefficient and the validity with the Confirmatory Factor Analysis from the WLSMV estimator. The invariance for various groups was reviewed with the Multigroup Confirmatory Factor Analysis, obtaining an Omega of .948. The studies performed allowed the demonstration of its reliability and validity with a factorial structure of a single factor. The multigroup analysis indicated invariance considering sex, developmental stages, schooling and place of residence. The analyses of the scores of the Cyberbullying Scale yielded evidence that it is reliable, valid and invariant in the Mexican population. The scale will provide reliable measures of cyberbullying to carry out studies that determine cause-effect relationships with other variables, as well as prevalence.
\end{abstract}

Keywords: information and communication technologies, anxiety, depression, victim, violence

\footnotetext{
${ }^{1}$ Doctor en Psicología. Profesor de Tiempo Completo. Departamento de Políticas Públicas, Universidad de Guadalajara. Periférico Norte, Núcleo Universitario Los Belenes, Zapopan, Jalisco, México, C.P. 45100. Tel.: +52(33)337703300. Correo: fcoqtapia@gmail.com

${ }^{2}$ Doctora en Ciencias Sociales con especialidad en Psicología Social. Profesora de Tiempo Completo. Facultad de Psicología, Universidad Michoacana de San Nicolás de Hidalgo. Francisco Villa, 450, Colonia Dr. Miguel Silva, Morelia, Michoacán, México C.P. 58110 . Tel.: +52 (44)33129909. Correo: maria.lourdes.vargas@umich.mx

Revista Iberoamericana de Diagnóstico y Evaluación - e Avaliação Psicológica. RIDEP · No62 · Vol.1 · 167-184 · 2022

ISSN: 1135-3848 print /2183-6051online
} 


\section{Introducción}

El ciberacoso es la conducta de uno o varios sujetos dirigida de manera intencionada $\mathrm{y}$ repetitiva para dañar a otro sujeto a través de las tecnologías de la información y la comunicación (TIC's): internet, celular, redes sociales, mensajería instantánea, entre otros (Sittichai \& Smith, 2018; Smith, 2016; Smith et al., 2008). En este sentido y de manera específica, los sujetos pueden hacer un uso indebido de las redes sociales e incidir en la perpetración de ciberacoso (Kircaburun, Kokkinos, Demetrovics, et al., 2019; Whittaker \& Kowalski, 2015).

Por otro lado, Kircaburun, Demetrovics, y Tosuntaş (2019) hicieron notar que el uso problemático de las redes sociales está asociado con el maquiavelismo (manipulación y engaño) y el narcisismo (comportamiento con extravagancia, arrogancia y grandiosidad), los cuales son factores que pueden incidir en el ciberacoso. Además, el uso intenso de las redes sociales se asocia con el desarrollo de síntomas psicopatológicos (Solano \& Perugini, 2019). Los usuarios de las redes sociales son susceptibles de experimentar un daño a partir de la distribución de contenido inapropiado personal y de otros; de ser rastreados sin su consentimiento e incluso, sin que sean conscientes de ello. Esto puede afectar su reputación con información falsa o verdadera, incluso, son susceptibles a perder el control de su identidad, así como a ser discriminados, excluidos y menospreciados por los grupos a los que pertenecen virtualmente (Baccarella et al., 2018).

El ciberacoso es un fenómeno considerado relativamente nuevo, que se deriva del acoso tradicional y se visualiza como un campo emergente con la necesidad de seguir profundizando sobre su conceptualización y formas de medirlo en el contexto adecuado, para captar las complejidades del fenómeno, las sutilezas tecnológicas y evitar hallazgos inconsistentes (Kofoed \& Staksrud, 2019; Olweus, 2012; Olweus \& Limber, 2018; Smith et al., 2012), por lo cual resulta relevante su estudio.

El ciberacoso y el acoso tradicional comparten cinco características principales: (a) existe un binomio perpetrador-víctima, (b) es un acto intencionado, (c) genera un daño, (c) implica un desequilibrio de poder y (d) es un acto que se repite (Campbell \& Bauman, 2018; Menesini et al., 2016; Smith, 2016). A pesar de que el ciberacoso y el acoso tradicional se encuentran altamente correlacionados, presentan diferencias operacionales significativas, como las que se describen a continuación.

Primeramente, en el ciberacoso, basta con realizar un único acto agresivo (forma directa) por parte del perpetrador para que sea reproducido varias veces por las demás personas (forma indirecta) y de esta manera sea experimentado en forma repetida por la víctima (Menesini et al., 2013). En segundo lugar, el desequilibrio de poder se resignifica, y se centra en las habilidades del agresor para el uso de tecnologías de la información y la comunicación, debido a que, al no haber contacto físico, la fuerza del agresor no es intimidante y sí lo es su potencialidad para agravar y difundir las agresiones verbales, psicológicas y relacionales (Barlett, 2019). En tercer lugar, se observa que en el ciberacoso, el perpetrador puede optar por el anonimato mientras que en el acoso tradicional el acto de agresión es cara a cara. En cuarto lugar, la víctima está accesible las 24 horas del día durante los siete días de la semana y existe mayor audiencia en los ataques (Kowalski et al., 2014). Finalmente, el ciberacoso puede considerarse una continuación o inicio del acoso tradicional, en una relación dialéctica de los espacios presenciales y virtuales donde se manifiesta el acto de dañar, de tal manera que permite al perpetrador continuar su comportamiento de acoso sobre la víctima (Chan et al., 2020; Chan \& Wong, 2016).

El ciberacoso se ha relacionado con distintos problemas de ámbitos diversos, tales como los psicosociales, los relacionados con la salud mental y/o con aspectos académicos; asimismo, se ha vinculado con ciertos rasgos de personalidad, tanto de la víctima como del perpetrador. De esta manera, el ciberacoso se asocia con mayores síntomas depresivos (Gamez-Guadix et al., 2013), mayor ansiedad (Gini et al., 2018), mayor hostilidad y agresión, baja autoestima, menor bienestar y satisfacción con la vida, mayor soledad, mayor uso indebido de sustancias, falta de compromiso moral, mayor comportamiento antisocial e hiperactividad (Kwan et al., 2020) y menor sentimiento de pertenencia a la escuela (Wright \& Wachs, 2019) ente otros. 
Muchas víctimas de ciberacoso han manifestado ideación suicida e intento suicida (Bauman et al., 2013; Hinduja \& Patchin, 2010, 2018; Van Geel et al., 2014). Asimismo, las víctimas que han sufrido simultáneamente ciberacoso y acoso tradicional, reportaron mayor daño en distintas áreas de la vida, a diferencia de quienes sufrieron solo alguna forma de acoso; aunque cabe hacer notar que, algunos autores como Alonso y Romero (2020); Cross et al. (2015); Schneider et al. (2012), encontraron que el ciberacoso tiene mayor efecto que el acoso tradicional.

El ciberacoso puede constituirse en un método más para dañar, como medio para tomar represalias por haber sido intimidado cara a cara o como un mecanismo para hacer y decir lo que en forma presencial no se realizaría (Kowalski \& Limber, 2013). Los problemas sociales y emocionales de los sujetos generan mayor probabilidad de ser víctimas o perpetradores tanto en el ciberacoso como en el acoso tradicional (Alonso \& Romero, 2020). Al respecto, Chen et al. (2017) reportaron como predictores de la perpetración de ciberacoso: el uso arriesgado de las tecnologías de la información y la comunicación, la desconexión moral, la depresión, las normas sociales, el acoso tradicional y el narcisismo.

Asimismo, Kowalski y Limber (2013) reportaron que en el ciberacoso y el acoso tradicional, las mujeres que intimidan presentaron mayores niveles de ansiedad y depresión con respecto a las mujeres que no participan en alguna forma de acoso; mientras que los hombres que intimidan, presentaron niveles similares de ansiedad y depresión en comparación con aquellos que no participan. También, Zych et al. (2018) reportaron que el perpetrador de ciberacoso se asocia con poca empatía cognitiva y afectiva combinadas, mientras que la víctima solo se relaciona con una alta empatía afectiva. Además, las mujeres tienen el doble de riesgo de ser víctimas de ciberacoso y acoso tradicional, son más propensas a informar y compartir con alguien, presentan menos problemas de conducta, mayores tendencias prosociales y más probabilidades de evitar la escuela (Alonso \& Romero, 2020; Kwan et al., 2020). Por otro lado, de acuerdo con Baier et al. (2019), los resultados de la medición de salud mental en víctimas de ciberacoso o acoso tradicional, indican mayor salud mental en los hombres que en las mujeres; no obstante, son los hombres quienes tienden a ser perpetradores de ciberacoso (Kircaburun, Kokkinos, Demetrovics, et al., 2019).

Para medir el ciberacoso se han desarrollado diversos instrumentos de evaluación. La mayoría de ellos son de tipo autoinforme, de un solo reactivo o multirreactivos, con escalas tipo Likert y dicotómicas. Hay instrumentos que difieren en la duración de los periodos en los que se exploran los eventos de ciberacoso en los sujetos: suelen medir las situaciones de ciberacoso vividas durante uno, dos, tres, cuatro, seis o 12 meses, previos a la encuesta; e incluso, hay algunos que no marcan un límite temporal, sino que consideran la experiencia global a lo largo de la vida del sujeto (Zych et al., 2016).

Se han desarrollado escalas separadas para medir la victimización y la perpetración, que consideran dichas variables como dos factores correlacionados. Tal es el caso del Cuestionario del Proyecto de Intervención del Ciberacoso Europeo (Del Rey et al., 2015, Ortega-Ruiz et al., 2016 Twardowska-Staszek et al., 2018) y del Cuestionario de Ciberacoso (Gamez-Guadix et al., 2014). Hay otros estudios que incluyeron el rol de observador como un factor más, tal es el caso de la Escala de Ciberacoso desarrollada por Capan et al. (2020) y de la Prueba de Ciberacoso propuesta por Garaigordobil (2015) o el factor Happy Slapping del Cuestionario de Ciberacoso reportado por Resett y Caino (2019) y obtuvieron una solución de tres factores correlacionados.

Existen también instrumentos que evalúan de manera separada la victimización y perpetración del ciberacoso, como la Escala de Perpetración del Ciberacoso y la Escala de Victimización del Ciberacoso desarrolladas por Lee et al. (2015); cada una de dichas escalas con una estructura factorial de segundo orden con tres factores de primer orden: Verbal/Escrita, Visual/Sexual y Exclusión Social. Otros, presentan tres factores correlacionados: Agresión Abierta, Relacional y Ciber, como el caso del Cuestionario del Proyecto Europeo de Intervención sobre Ciberacoso con evidencias de validación para población residente en Estados Unidos (Williford 
\& DePaolis, 2019). Algunos más, consideran cuatro factores correlacionados: Física, Verbal, Relacional y Ciber de la Escala de Acoso y Ciberacoso para Adolescentes desarrollada por Thomas et al. (2019).

Asimismo, se han desarrollado instrumentos para medir solo la victimización. Al respecto, se han reportado estructuras de un solo factor, como el Cuestionario de Cibervictimización (ÁlvarezGarcía et al., 2015) y las Escalas Multireactivos para medir el Ciberacoso (Fluck, 2018); otras, con dos factores correlacionados: acoso a través del teléfono móvil y acoso a través de internet, como la Escala de Victimización entre Adolescentes a través del Teléfono Móvil y de Internet, desarrollada por Buelga et al. (2012) y con evidencias de validez en estudiantes chilenos por Galvez-Nieto et al. (2016). Además, se encontraron algunas escalas con tres factores correlacionados: Personal, Intrusión y Relacionados al Trabajo, tal es el caso del Inventario de Actos de Ciberacoso en el Trabajo (Vranjes et al., 2017); y algunos más con cuatro factores correlacionados: Directamente en Línea, Indirectamente en Línea, Directamente Desconectado, Indirectamente Desconectado, como por ejemplo, la Escala de Victimización entre Pares Fuera de Línea y en Línea (HaidStecher et al., 2019). Todos los estudios han reportado evidencias de confiabilidad en los instrumentos.

En medio de esta diversidad de instrumentos se encuentra una discusión acerca de las ventajas y desventajas para cada elemento de los instrumentos. Sin embargo, existe consenso en que la diversidad en los instrumentos para medir el ciberacoso tiene dos consecuencias: (a) la imposibilidad de comparación entre prevalencias, debido a las variaciones que se reportan; (b) las mediciones en las relaciones del ciberacoso pueden variar al asociarlas con otras variables. Asimismo, se encontró que los instrumentos multirreactivos han presentado evidencias de mayor validez y confiabilidad, por lo que son los más empleados para verificar la presencia de comportamientos de ciberacoso (Kowalski et al., 2014; Thomas et al., 2014). La medición del ciberacoso durante periodos mayores permite valorar prevalencia a lo largo de la vida; y los instrumentos que valoran las intervenciones sobre el ciberacoso, son mejores cuando miden periodos más cortos (Zych et al., 2016).

En el contexto mexicano, Buelga et al. (2012) presentaron evidencias de validez de la Escala de Victimización entre Adolescentes a través del Teléfono móvil y de Internet con una muestra de 309 estudiantes adolescentes de Sinaloa, México. Por otro lado, Gamez-Guadix et al. (2014) presentaron evidencias de validez de un Cuestionario de Ciberacoso en Estudiantes Adolescentes de la Ciudad de México, en el que se evalúa la perpetración y victimización, y reportaron que la muestra no es representativa de la población mexicana. Como puede apreciarse, ambos instrumentos fueron analizados en zonas específicas de México, por lo que ninguno de ellos contó con una muestra representativa del país. El estudio más relevante sobre el tema, ha sido el realizado por el Instituto Nacional de Estadística y Geografía (INEGI) en México, a través del Módulo sobre Ciberacoso (MOCIBA) (INEGI, 2017a), ya que evalúa a gran escala el Ciberacoso en toda la República Mexicana.

En el MOCIBA, se encuentran diez reactivos que valoran las situaciones de ciberacoso experimentadas por la víctima a través de los medios electrónicos como: recibir mensajes y llamadas ofensivas, publicación de información personal para dañar, recibir críticas en relación con la apariencia o clase social, recibir insinuaciones de tipo sexual y usurpación de la identidad para enviar información falsa. El instrumento MOCIBA se centra en la evaluación del rol de víctima, como sucede con el Cuestionario de Cibervictimización (Álvarez-García et al., 2015), la Escala de Victimización entre Adolescentes a través del Teléfono Móvil y de Internet (Buelga et al., 2012; Galvez-Nieto et al., 2016) y las Escalas Multireactivo para medir Ciberacoso (Fluck, 2018); por lo que se diferencia del Cuestionario del Proyecto Europeo de Intervención sobre Ciberacoso (Del Rey et al., 2015; Ortega-Ruiz et al., 2016; Twardowska-Staszek et al., 2018) y del Cuestionario de Ciberacoso (Gamez-Guadix et al., 2014). No obstante, a pesar de haberse aplicado a una población considerable, no se han reportado las propiedades psicométricas de los reactivos que se utilizan en el MOCIBA.

Por otro lado, existen pocos estudios en los que se demuestre que los instrumentos de gran 
escala tienen las propiedades psicométricas adecuadas y que permitan comparar los diferentes grupos poblacionales tanto geográficos como por sus características sociodemográficas. El MOCIBA es un Módulo de Ciberacoso de la Encuesta Nacional sobre Disponibilidad y Uso de las TIC en Hogares (ENDUTIH), que está destinado a recuperar información sobre el fenómeno de ciberacoso en la población mexicana de 12 años y más, es de utilidad para los investigadores interesados en el tema y puede dar pie para generar políticas públicas en México. El MOCIBA se ha aplicado durante los años 2015, 2016, 2017, 2019 y 2020, y recupera información sobre las situaciones experimentadas de ciberacoso, la frecuencia y el medio en el que se experimentó, el sexo, identidad y motivación de la persona acosadora, los efectos en la víctima y las medidas adoptadas frente la situación de ciberacoso experimentada. Por otra parte, el MOCIBA fue construido ad hoc por equipos de trabajo interno del INEGI para el estudio del ciberacoso en la población mexicana, a partir de la revisión y análisis de diversos estudios prácticos y teóricos, encuestas, preguntas, variables $\mathrm{y}$ clasificaciones del ciberacoso a nivel nacional e internacional, poniendo mayor énfasis en aquellos reportes que propusieron un listado de diferentes tipos de ciberacoso, con la finalidad de seleccionar las más representativas y las que reportaron mayor frecuencia de ciberacoso. En consecuencia, es importante que el MOCIBA, que se utiliza a escala nacional, cuente con las evaluaciones de las propiedades psicométricas de las puntuaciones a los reactivos centrales que evalúan el Ciberacoso, ya que se requiere dar cuenta de las evidencias de validez y confiabilidad que tienen los reactivos para la población mexicana. De este modo, al aplicar el MOCIBA, se obtiene evidencia de que los reactivos permiten dar cuenta del constructo teórico de Ciberacoso y lo miden de manera consistente y precisa dentro de la población mexicana.

Asimismo, consideramos necesario realizar pruebas de invarianza a los reactivos del MOCIBA que valoran las situaciones de ciberacoso experimentadas por la población mexicana, agrupándola por sexo, estadios del desarrollo humano, nivel de estudios y lugar de residencia. De esta manera, y con base en lo propuesto por Cheung y Rensvold (2002), obtenemos evidencias de invarianza en distintos niveles de las puntuaciones de los participantes a la Escala de Ciberacoso: (a) invarianza configuracional, los constructos se conceptualizan de la misma manera por sujetos de distintos grupos; (b) invarianza métrica, los pesos factoriales de todos los parámetros del modelo son iguales en todos los grupos comparados; (c) invarianza escalar, la escala de medición es conceptualizada operativamente de la misma manera en todos los grupos.

Diversos autores recomiendan fortalecer el trabajo acerca de la conceptualización y formas de evaluación del ciberacoso, además de analizar las propiedades psicométricas de los instrumentos, preferentemente con métodos de análisis factorial confirmatorio para indagar diferentes soluciones factoriales, así como valorar la invarianza en distintos grupos poblacionales (Kowalski et al., 2014; Thomas et al., 2014; Zych et al., 2016). De igual modo, en los estudios revisados se ha recomendado ampliar las muestras para lograr la representatividad de los resultados, así como incluir otros grupos poblacionales distintos a los estudiantes. Todo lo antes mencionado, permite evidenciar la conveniencia de analizar las propiedades psicométricas de una Escala de Ciberacoso para la población mexicana, a partir de las puntuaciones de algunos reactivos del MOCIBA, lo cual constituye el objetivo del presente artículo.

\section{Método}

\section{Participantes}

La muestra quedó conformada por 33289 participantes de los 32 Estados de la República Mexicana: 16307 (49\%) hombres y 16982 (51\%) mujeres. El rango de edad reportado fue de 12 a 59 años $(M=31, D E=12)$, de modo tal que: 6961 (20.9\%) corresponden al rango de 12 a 19 años; 9523 (8.6\%), de 20 a 29 años; 7455 (22.4\%), de 30 a 39 años; 5578 (16.8\%), de 40 a 49 años; y 3772 (11.3\%), de 50 a 59 años. De acuerdo con el Estadio del Desarrollo Humano de los participantes, 26328(79\%) fueron adultos y 6961(21\%) fueron adolescentes. Asimismo, en la Tabla 1 se presentan las características de la muestra por nivel de estudios y lugar de residencia. 
Tabla 1. Características sociodemográficas los participantes

\begin{tabular}{|c|c|c|}
\hline Características & $\mathrm{n}$ & $\%$ \\
\hline \multicolumn{3}{|l|}{ Nivel de estudios } \\
\hline Ninguno & 99 & 0.3 \\
\hline Educación Básica & 14325 & 43.3 \\
\hline Preescolar o kínder & 5 & 0.01 \\
\hline Primaria & 3701 & 11.1 \\
\hline Secundaria & 9964 & 29.9 \\
\hline Normal básica & 61 & 0.2 \\
\hline Técnico terminal con secundaria & 594 & 1.8 \\
\hline \multicolumn{3}{|l|}{ Educación Media Superior } \\
\hline Preparatoria o bachillerato & 8251 & 24.9 \\
\hline Educación Superior & 10535 & 31.8 \\
\hline Técnico superior con preparatoria terminada & 1029 & 3.1 \\
\hline Licenciatura o ingeniería & 8508 & 25.6 \\
\hline Especialidad & 188 & 0.6 \\
\hline Maestría & 702 & 2.1 \\
\hline Doctorado & 108 & 0.3 \\
\hline No sabe & 79 & 0.2 \\
\hline \multicolumn{3}{|l|}{ Lugar de residencia: Región del País/Estado } \\
\hline Región Norte & 13216 & 39.7 \\
\hline Baja California & 1939 & 5.8 \\
\hline Sonora & 1361 & 4.1 \\
\hline Chihuahua & 1232 & 3.7 \\
\hline Coahuila de Zaragoza & 1080 & 3.2 \\
\hline Nuevo León & 1052 & 3.2 \\
\hline Tamaulipas & 2,068 & 6.2 \\
\hline Baja California Sur & 889 & 2.7 \\
\hline Sinaloa & 1256 & 3.8 \\
\hline Nayarit & 783 & 2.4 \\
\hline Durango & 828 & 2.5 \\
\hline Zacatecas & 728 & 2.2 \\
\hline Región Centro & 12471 & 37.5 \\
\hline Jalisco & 1072 & 3.2 \\
\hline Aguascalientes & 796 & 2.4 \\
\hline Colima & 853 & 2.6 \\
\hline Michoacán de Ocampo & 1244 & 3.7 \\
\hline San Luis Potosí & 686 & 2.1 \\
\hline Guanajuato & 1703 & 5.1 \\
\hline Querétaro & 791 & 2.4 \\
\hline Hidalgo & 734 & 2.2 \\
\hline Estado de México & 1221 & 3.7 \\
\hline Ciudad de México & 817 & 2.5 \\
\hline Morelos & 743 & 2.2 \\
\hline Tlaxcala & 664 & 2.0 \\
\hline Puebla & 1147 & 3.4 \\
\hline Región Sur & 7602 & 22.8 \\
\hline Guerrero & 1031 & 3.1 \\
\hline Oaxaca & 709 & 2.1 \\
\hline Chiapas & 1079 & 3.2 \\
\hline Veracruz & 1573 & 4.7 \\
\hline Tabasco & 763 & 2.3 \\
\hline Campeche & 756 & 2.3 \\
\hline Quintana Roo & 851 & 2.6 \\
\hline Yucatán & 840 & 2.5 \\
\hline
\end{tabular}

\section{Instrumento}

En el año 2017, Instituto Nacional de Estadística y Geografía (INEGI, 2017a), aplicó la Encuesta Nacional sobre Disponibilidad y Uso de las Tecnologías de la Información en los Hogares (ENDUTIH). Uno de los módulos de dicha encuesta, denominado Módulo de Ciberacoso (MOCIBA), está formado por un total de 167 preguntas que recuperan información sobre las variables siguientes: (a) condición de uso de medidas de seguridad, (b) medidas de seguridad realizadas, (c) condición de recepción de correo basura o virus, (d) condición de haber experimentado ciberacoso, (e) percepción de protección de datos, (f) situaciones de ciberacoso experimentadas, (g) identidad de la persona acosadora, (h) sexo de la persona acosadora, (i) motivación de la persona acosadora, (j) frecuencia 
de ciberacoso, (k) efectos en la víctima y (l) medidas contra el ciberacoso experimentado. De las 167 preguntas, seleccionamos los 10 reactivos que evalúan las situaciones de ciberacoso experimentadas por los participantes en el año previo a la encuesta. Tales reactivos coinciden con los que se han reportado en la literatura para evaluar la victimización del ciberacoso como un solo factor. En el instrumento, los participantes deben responder Sí, No o No Sé a cada reactivo (ver Anexo)

\section{Procedimiento}

De acuerdo con el INEGI (2017b, 2017c), los entrevistadores fueron capacitados de manera presencial y a distancia; la muestra fue recuperada de manera probabilística, polietápica, estratificada y por conglomerados en los 32 estados de la República Mexicana. El trabajo de campo se realizó del 29 de mayo al 21 de julio de 2017; y la encuesta se realizó de forma presencial, en papel o con dispositivos electrónicos, en los domicilios de los participantes que cumplieron los criterios de ser sujetos de 12 a 59 años y usuarios de internet en los últimos tres meses anteriores a la aplicación de la encuesta.

Recuperamos la base de datos que contiene las respuestas al cuestionario MOCIBA $2017 \mathrm{y}$ que fue publicada por el INEGI (2017d) en su portal electrónico, aprovechando la situación de que la información generada por el INEGI es de carácter público, gratuito y de libre uso, y se puede publicar, adaptar, reordenar, explotar comercialmente y extraer total o parcialmente (Ley del Sistema Nacional de Información Estadística y Geografía de 2018; Norma para la difusión y promoción del acceso, conocimiento y uso de la información estadística y geográfica que genera el Instituto Nacional de Estadística y Geografía de 2018; Términos de libre uso de la Información del Instituto Nacional de Estadística y Geografía de 2019).

De la base de datos, eliminamos 277 casos que respondieron a los reactivos con No sé, debido a que se busca validar los reactivos con una escala dicotómica (Sí, No).

\section{Análisis estadísticos}

Para los análisis descriptivos de la muestra empleamos la Tabla de frecuencias. Utilizamos el Coeficiente Omega $(\omega)$ para obtener evidencia de la fiabilidad la escala. Para reportar evidencias de validez de constructo aplicamos el Análisis Factorial Confirmatorio (AFC) con el método de weighted least square mean and variance adjusted (WLSMV). Evaluamos el modelo de un solo factor debido a que los 10 reactivos seleccionados del MOCIBA que evalúan las situaciones de ciberacoso experimentadas coinciden teóricamente con el ciberacoso desde el rol de la víctima como un solo constructo. Las medidas de evaluación fueron: chi-cuadrado $\left(\chi^{2}\right)$, grados de libertad $(g l)$, residuo estandarizado cuadrático medio (SRMR), error cuadrático medio de aproximación (RMSEA), índice de ajuste comparativo (CFI), y el índice de Tuker-Lewis (TLI).

Elegimos el estimador WLSMV, debido a que permite el análisis de la matriz de relaciones tetracóricas para datos categóricos binarios (Flora \& Curran, 2004; Liang \& Yang, 2014; Rhemtulla et al., 2012). Asimismo, para evaluar la invarianza por grupos de sexo, estadio del desarrollo, nivel de estudios y lugar de residencia utilizamos el Análisis Factorial Multigrupo (AFC-MG), con los modelos que recomienda Vandenberg y Lance (2000): invarianza de configuración, invarianza métrica e invarianza escalar. Además, realizamos el análisis de la invarianza por grupos del lugar de residencia con el método de alineación, debido a que este método nos permite hacer un análisis factorial confirmatorio de múltiples grupos con la capacidad de estimar modelos para muchos grupos, se basa en el modelo de configuración y arroja un análisis detallado de la invarianza para cada parámetro del modelo en cada grupo (Muthén \& Asparouhov, 2014).

Para dar evidencia de la confiabilidad de la escala asignamos como criterio un valor del coeficiente omega mayor o igual a .75 (McDonald, 1999). Para interpretar como bueno el ajuste de los datos al modelo de la escala propuesto en el AFC establecimos los valores de SRMR $\leq .08, \quad$ RMSEA $\leq .06, \quad$ CFI $\geq .95$ y TLI $\geq .95$ (Hu \& Bentler, 1999). Para analizar la invarianza de la escala en los distintos grupos por sexo (hombre y mujer), por lugar de residencia de acuerdo con la región del país (norte, centro y sur) $\mathrm{y}$ por estadios del desarrollo humano (adolescencia y adulto) y nivel de estudios 
(educación básica, media superior y superior), empleamos el criterio de cambio de $\Delta$ CFI <-.01 (Cheung \& Rensvold, 2002). Para dar evidencia de la confiabilidad de los resultados del método de alineación establecimos un límite del 25\% de noinvarianza para los parámetros de interceptos y las cargas de los reactivos (Muthén \& Asparouhov, 2014). Por último, utilizamos el programa informático de SPSS 25 para los análisis descriptivos y Mplus 8.6 para el análisis de coeficiente omega, validez factorial e invarianza con el estimador WLSMV y el método de alineación.

\section{Resultados}

\section{Análisis Factorial Confirmatorio}

El modelo con una estructura factorial de un solo factor, presenta un buen ajuste con los datos de la Escala de Ciberacoso de la población mexicana. El AFC arrojó los siguientes valores de los índices: $\chi^{2}=1552 \quad(p<.05), \quad g l=35$, RSMEA $=.036$ [90\% IC; .035, .038], SRMR $=.05$, $\mathrm{CFI}=.98, \mathrm{TLI}=.97$. El valor de chi-cuadrado indica rechazar el ajuste de los datos con el modelo; sin embargo, esto es común para muestras grandes, por lo que la literatura sugiere determinar el ajuste con el análisis de los demás índices presentados (Bentler \& Bonett, 1980; Hu \& Bentler, 1998).

Los valores de los índices de RMSEA, SRMR, CFI y TLI indican un buen ajuste del modelo y los datos de la presente investigación, lo anterior con base en los puntos de corte establecidos. De esta manera, los diez reactivos observados en los participantes del estudio y que recuperan las situaciones de ciberacoso experimentadas permiten ser explicados por un factor latente denominado Ciberacoso. Además, los diez reactivos que conforman la Escala de Ciberacoso en México presentan pesos factoriales estandarizados superiores a .70 (ver Tabla 2). Por lo tanto, los análisis de las puntuaciones a los reactivos de la Escala de Ciberacoso arrojaron evidencia de validez de constructo para la población de México.

\section{Análisis de Invarianza}

Los análisis de las puntuaciones a la Escala de Ciberacoso aplicada en México arrojaron evidencia de invarianza para la población agrupada por sexo: hombres y mujeres; estadios del desarrollo: adolescentes y adultos; nivel de estudios: educación básica, media y superior; y región de residencia en el País: norte, centro y sur. La Tabla 3 muestra los índices de ajuste para los modelos del Análisis Factorial Confirmatorio por grupos separados y el Análisis Factorial Confirmatorio Multigrupo para los modelos configuracionales y escalares por los grupos de referencia. Los AFC por grupos separados por sexo, estadios del desarrollo, nivel de estudios y región de residencia en el País arrojaron valores en los índices de RMSEA, SRMR, TLI y CFI que sugieren un buen ajuste entre las puntuaciones a los reactivos y el modelo de un solo factor para la Escala de Ciberacoso en la población mexicana. Por lo tanto, se sugiere evidencia de invarianza en las puntuaciones de la Escala de Ciberacoso aplicada en los distintos grupos de análisis de la población de México.

Asimismo, en la Tabla 3 se aprecia que los valores de CFI que corresponden a los modelos configuracionales son mayores a .95 para todos los grupos comparados, lo que indica que se deben aceptar los modelos base de invarianza configuracional. Además, el modelo de invarianza métrica no converge con el estimador WLSMV para datos estadísticos binarios. Por su parte, los valores del $\triangle \mathrm{CFI}$ entre el modelo configuracional y el modelo escalar de los grupos en referencia tiene como resultado de .00; lo anterior sugiere evidencia de equivalencia de interceptos en todos grupos comprados.

Por otra parte, se aplicó el método de alineación para valorar la invarianza por grupos de lugar de residencia. En la Tabla 4 se observa la no-invarianza del reactivo uno en el Estado de Zacatecas (32); del reactivo seis en el estado de Aguascalientes (1); y del reactivo ocho en el estado de Sinaloa (25). Asimismo, se observa que los demás parámetros de los reactivos son significativamente invariantes en los distintos grupos de análisis. De esta manera, se obtiene que solo $9.3 \%$ de los interceptos son no-invariantes. Lo anterior indica evidencia de confiabilidad en los resultados de alineación, debido a que se estableció como límite del $25 \%$ para valorar la evidencia de la confiabilidad del análisis. Por lo tanto, los análisis de las puntuaciones a los diez 
Tabla 2. Pesos factoriales de los reactivos en el Análisis Factorial Confirmatorio

\begin{tabular}{cll}
\hline \multicolumn{1}{c}{ Reactivos } & \multicolumn{1}{c}{ Pesos Factoriales } \\
\hline De junio de 2016 a la fecha, dígame si ¿por teléfono celular o internet alguien... & .85 \\
1 & le envió mensajes ofensivos, con insultos o burlas? & .73 \\
2 & le hizo llamadas ofensivas, con insultos o burlas? & .75 \\
3 & publicó información personal, fotos o videos (falsos o verdaderos) de usted para dañarlo(a)? & .79 \\
4 & lo(a) criticó o se burló de usted en línea por su apariencia o clase social? & .79 \\
5 & le hizo insinuaciones o propuestas de tipo sexual? & .75 \\
6 & se hizo pasar por usted para enviar información falsa, insultar o agredir a otros? & .85 \\
7 & lo(a) contactó con nombres falsos para molestarle o dañarle? & .86 \\
8 & vigiló sus sitios o cuentas en Internet para causarle molestia o daño? & .86 \\
9 & lo(a) provocó (molestó o retó) en línea para que reaccione de forma negativa? & .77 \\
10 & le envió fotos o videos de contenido sexual para molestarle? & \\
\hline
\end{tabular}

Nota. Todos los coeficientes son significativos $(p<.05)$.

Tabla 3. Índices de ajuste de la prueba de invarianza de la Escala de Ciberacoso para distintos grupos

\begin{tabular}{|c|c|c|c|c|c|c|c|}
\hline \multirow{2}{*}{ Grupos/Modelos ${ }^{\text {a }}$} & \multicolumn{7}{|c|}{ WLSMV } \\
\hline & $X$ & $g l$ & RMSEA [90\% IC] & SRMR & TLI & CFI & $\Delta \mathrm{CFI}$ \\
\hline \multicolumn{8}{|l|}{ Sexo } \\
\hline \multicolumn{8}{|l|}{ Fase 1. Analisis Factorial } \\
\hline \multicolumn{8}{|l|}{ Confirmatorio } \\
\hline Hombres $(n=16,307)$ & $608 *$ & 35 & $.032[.030, .034]$ & .05 & .98 & .98 & - \\
\hline Mujeres $(n=16,982)$ & $876^{*}$ & 35 & $.038[.035, .040]$ & .05 & .97 & .98 & - \\
\hline \multicolumn{8}{|l|}{ Fase 2. Analisis Factorial Multigrupo } \\
\hline Configuracional & $1493^{*}$ & 70 & $.035[.033, .037]$ & .05 & .97 & .98 & - \\
\hline Escalar & $1625^{*}$ & 78 & $.035[.033, .036]$ & .05 & .97 & .98 & .00 \\
\hline \multicolumn{8}{|l|}{ Estadios del Desarrollo } \\
\hline \multicolumn{8}{|l|}{ Fase 1. Analisis Factorial } \\
\hline \multicolumn{8}{|l|}{ Confirmatorio } \\
\hline Adolescentes $(n=6,961)$ & $354 *$ & 35 & $.036[.033, .040]$ & .05 & .97 & .97 & - \\
\hline Adultos $(n=26,328)$ & $1232 *$ & 35 & $.036[.034, .038]$ & .05 & .97 & .98 & - \\
\hline \multicolumn{8}{|l|}{ Fase 2. Analisis Factorial Multigrupo } \\
\hline Configuracional & $1551^{*}$ & 70 & $.036[.034, .037]$ & .05 & .97 & .98 & - \\
\hline Escalar & $1392 *$ & 78 & $.032[.030, .033]$ & .05 & .98 & .98 & .00 \\
\hline \multicolumn{8}{|l|}{ Nivel de estudios ${ }^{b}$} \\
\hline \multirow{2}{*}{\multicolumn{8}{|c|}{ Fase 1. Analisis Factorial }} \\
\hline Confirmatorio & & & & & & & \\
\hline Educación Básica $(n=14,325)$ & $610^{*}$ & 35 & $.034[.032, .036]$ & .05 & .97 & .98 & - \\
\hline $\begin{array}{l}\text { Educación Media Superior }(n= \\
8,251)\end{array}$ & $542 *$ & 35 & $.042[.039, .045]$ & .06 & .96 & .97 & - \\
\hline Educación Superior $(n=10,535)$ & $454^{*}$ & 35 & $.034[.031, .037]$ & .05 & .98 & .98 & - \\
\hline \multicolumn{8}{|l|}{ Fase 2. Analisis Factorial Multigrupo } \\
\hline Configuracional & $1609 *$ & 105 & $.036[.034, .038]$ & .05 & .97 & .98 & - \\
\hline Escalar & $1417 *$ & 121 & $.031[.030, .033]$ & .05 & .98 & .98 & .00 \\
\hline \multicolumn{8}{|l|}{ Región de residencia en el País b } \\
\hline \multicolumn{8}{|l|}{ Fase 1. Analisis Factorial } \\
\hline \multicolumn{8}{|l|}{ Confirmatorio } \\
\hline Norte $(n=13,216)$ & $640 *$ & 35 & $.036[.034, .039]$ & .05 & .97 & .98 & - \\
\hline Centro $(n=12,471)$ & $597 *$ & 35 & $.036[.033, .038]$ & .05 & .97 & .98 & - \\
\hline $\operatorname{Sur}(n=7,602)$ & $357 *$ & 35 & $.035[.032, .038]$ & .05 & .97 & .97 & - \\
\hline \multicolumn{8}{|l|}{ Fase 2. Analisis Factorial Multigrupo } \\
\hline Configuracional & $1585^{*}$ & 105 & $.036[.034, .037]$ & .05 & .97 & .98 & - \\
\hline Escalar & $1328 *$ & 121 & $.030[.029, .031]$ & .05 & .98 & .98 & .00 \\
\hline
\end{tabular}

Nota. WLSMV=estimación de mínimos cuadrados ponderados con media y varianza ajustada; $\mathrm{ML}=$ máxima verosimilitud; $\chi^{2}=$ chi-cuadrado; $g l=$ grados de libertad; RMSEA=error cuadrático medio de aproximación; IC=intervalo de confianza; SRMR=residuo estandarizado cuadrático medio; TLlíndice de Tuker Lewis; $\mathrm{CFI}=$ ndice de ajuste comparative; $\Delta \mathrm{CFI}=$ cambio en el valor del índice de ajuste comparativo.

${ }^{a}$ La invarianza métrica para variables dicotómicas no converge con el estimador WLSMV. ${ }^{\text {b }}$ El desglose de los niveles educativos y los Estados de la República Mexicana que constituyen las regiones de residencia se muestran en el apartado de participantes. * $p<.05$

reactivos que conforman la Escala de Ciberacoso arrojaron evidencias de que son invariantes para los grupos de lugar de residencia de la población mexicana.

\section{Fiabilidad}

El análisis del coeficiente Omega arrojó un valor de .948 [95\% IC=.945; .951]. Por lo tanto, el análisis de confiabilidad de las puntuaciones a la escala de Ciberacoso aplicada en México arrojó evidencia de ser confiable. 
Tabla 4. Medida aproximada (No)Invarianza para interceptos y cargas por lugar de residencia

\begin{tabular}{ll}
\hline Reactivo & Interceptos \\
\hline 1 & 12345678910111213141516171819202122232425262728293031 (32) \\
2 & 1234567891011121314151617181920212223242526272829303132 \\
3 & 1234567891011121314151617181920212223242526272829303132 \\
4 & 1234567891011121314151617181920212223242526272829303132 \\
5 & 1234567891011121314151617181920212223242526272829303132 \\
6 & $(1) 234567891011121314151617181920212223242526272829303132$ \\
7 & 1234567891011121314151617181920212223242526272829303132 \\
8 & $123456789101112131415161718192021222324(25) 26272829303132$ \\
9 & 1234567891011121314151617181920212223242526272829303132 \\
10 & 1234567891011121314151617181920212223242526272829303132 \\
1 & Cargas \\
2 & 1234567891011121314151617181920212223242526272829303132 \\
3 & 1234567891011121314151617181920212223242526272829303132 \\
4 & 1234567891011121314151617181920212223242526272829303132 \\
5 & 1234567891011121314151617181920212223242526272829303132 \\
6 & 1234567891011121314151617181920212223242526272829303132 \\
7 & 1234567891011121314151617181920212223242526272829303132 \\
8 & 1234567891011121314151617181920212223242526272829303132 \\
10 & 1234567891011121314151617181920212223242526272829303132 \\
\hline
\end{tabular}

Nota. Los números en paréntesis refieren a los lugares de residencia que muestran significante no-invarianza por los parámetros. Aguascalientes (1), Baja California (2), Baja California Sur (3), Campeche(4), Coahuila de Zaragoza (5), Colima (6), Chiapas (7), Chihuahua (8), Ciudad de México (9), Durango (10), Guanajuato (11), Guerrero (12), Hidalgo (13), Jalisco (14), Estado de México (15), Michoacán de Ocampo (16), Morelos (17), Nayarit (18), Nuevo León (19), Oaxaca (20), Puebla (21), Querétaro (22), Quintana Roo (23), San Luis Potosí (24), Sinaloa (25), Sonora (26), Tabasco (27), Tamaulipas (28), Tlaxcala (29), Veracruz (30), Yucatán (31), Zacatecas (32).

\section{Discusión}

El objetivo del presente trabajo fue analizar las propiedades psicométricas de una Escala de Ciberacoso para población mexicana. Los análisis realizados sugieren evidencia de confiabilidad y validez de constructo de la Escala en México, construida por diez reactivos de respuesta dicotómica del MOCIBA, con una estructura factorial de un solo factor latente. Asimismo, los análisis de las puntuaciones a la Escala de Ciberacoso arrojaron evidencia de ser equivalentes entre los grupos poblaciones por sexo, estadio del desarrollo humano, nivel de estudios y lugar de residencia en México.

La estructura factorial de los instrumentos que evalúan ciberacoso es diversa. Nuestro estudio arrojó evidencia de que la Escala de Ciberacoso en población mexicana presenta una estructura de un solo factor que recupera las experiencias subjetivas de la víctima, como se ha demostrado en el estudio con el Cuestionario de
Cibervictimización (Álvarez-García et al., 2015). Aunque se han encontrado invetigaciones en las que tambien se evaluó solo el rol de la victíma en el ciberacoso, nuestros estudios difieren de los obtenidos en dichas investigaciones, tanto en lo que se refiere a los medios utilizados, como en los espacios y formas en que se dio la situación.

En primer lugar, hubo diferencias con los resultados de la Escala de Victimización entre Adolescentes a través del Teléfono Móvil y de Internet (Buelga et al., 2012; Galvez-Nieto et al., 2016) debido a que las experiencias de la víctima se evaluaron separando los medios a través de los cuales se ejecutó la agresión: el internet y/o el teléfono móvil. En segundo lugar, se distinguen de la investigación con la Escala de Victimización entre Pares Fuera de Línea y en Línea (HaidStecher et al., 2019), debido a que la evaluación de la forma de victimización se realizó de acuerdo con el espacio en el que se experimentó la agresión: en línea o presencial; y si la agresión ha sido de manera directa mediante conductas de 
insulto, mensajes, ofensivos y desagradables, o de forma indirecta a partir de ignorar y excluir a la víctima en las conversaciones o actividades grupales. En tercer lugar, difiere con lo reportado en el estudio con el Inventario de Actos de Ciberacoso en el Trabajo (Vranjes et al., 2017), debido a que los autores exploraron las experiencias en el entorno laboral, ya sea de situaciones relacionadas con el trabajo, relacionadas con la persona o en casos de intrusión. Las diferencias encontradas con las investigaciones referidas son esperadas, debido a que dichos insrumentos recuperaron un contenido más amplio de las situaciones de ciberacoso experimentadas por la víctima en terminos de medios y formas de ejecución de la agresión recibida: si fue por internet, teléfono móvil, si estuvieron en línea o no, ya sea de manera directa $o$ indirecta o si fue en el contexto laboral o escolar.

Asimismo, en relación con las escalas desarrolladas por separado para evaluar los roles de víctima y de perpetrador, nuestro estudio difiere con lo reportado por Lee et al. (2015) en la Escala de Victimización por Ciberacoso, debido a que ellos reportaron la escala con una estructura factorial de segundo orden con tres factores: Verbal/Escrita, Visual/Sexual y Exclusión Social. Difiere también con Williford y DePaolis (2019), en la modificación del Cuestionario del Proyecto Europeo de Intervención sobre Ciberacoso, debido a que elaboraron reactivos para las situaciones de agresion abierta, de tipo relacional y medios de ejecución en el ciberespacio. De esta manera, hicieron los reactivos comprensibles para los estudiantes de primaria, y así lograron una estructura de tres factores correlacionados. $\mathrm{Y}$ en nuestro estudio, cada reactivo evalúa la situación de acoso y medio electrónico de ejecución en el ciberespacio, lo que permitió obtener una estructura de un solo factor. Las diferencias encontradas también son esperadas, debido a que los instrumentos recuperaron contenidos diversos sobre el rol de la víctima en el ciberacoso y dichos contenidos reflejan diferencias estructurales en términos factoriales.

También nuestros resultados son diferentes con los reportados por Thomas et al. (2019) sobre la Escala de Acoso y Ciberacoso para Adolescentes, ya que manejaron una estructura de tres factores correlacionados: Física, Verbal, Relacional y Ciberespacio, lo que permite valorar de manera simultánea el acoso tradicional y el ciberacoso. Sin embargo, nuestros resultados coinciden con Thomas y colaboradores específicamente con la Escala de Ciberespacio, ya que se evalúan las experiencias de acoso de la víctima a través del celular o de estar en línea, en un solo factor, como lo hace nuestra escala en la población mexicana.

Por otro lado, la literatura sobre los instrumentos para medir el ciberacoso reportó datos de confiabilidad tanto para la escala global como para cada uno de los factores analizados. Nuestro estudio coincide con lo reportado por la literatura, en términos de confiabilidad de la escala para evaluar la victimización en el contexto del ciberespacio (cfr. Álvarez-García et al., 2015; Buelga et al., 2012; Capan et al., 2020; Del Rey et al., 2015; Garaigordobil, 2015; Resett \& GamezGuadix, 2018; Twardowska-Staszek et al., 2018). Sin embargo, solo se encontraron diferencias de nuestros resultados de confiabilidad con los demás estudios mencionados, en el número total de reactivos y las escalas utilizadas en cada instrumento.

Esta investigación presenta limitaciones que se constituyen como áreas de oportunidad de desarrollo científico en la línea de estudio del ciberacoso. En este sentido, en este estudio solo se analiza la validez de constructo de la Escala de Ciberacoso aplicada en población mexicana. Además, los reactivos de la escala evalúan únicamente las experiencias subjetivas de ciberacoso de la víctima, de tal manera, que no se cubre todo el espectro teórico que involucra el ciberacoso. Asimismo, la Escala de Ciberacoso se construyó ad hoc para fines estadísticos del INEGI, a partir de la recuperación de los reactivos que diversos instrumentos internacionales emplean para medir el ciberacoso, sin considerar las pautas internacionales para la construcción de test. Otra de las limitaciones consiste en que el instrumento es un autoinforme y la deseabilidad social puede influir en las respuestas.

Por lo tanto, al aplicar la Escala de Ciberacoso, se sugiere ser cautelosos en las interpretaciones que de ella se deriven. Asimismo, se sugiere en futuras investigaciones: (a) obtener evidencias de validez convergente, predictiva y 
discriminante de la Escala de Ciberacoso, (b) redactar los reactivos que recuperen las situaciones de ciberacoso desde el rol del perpetrador y del observador para tener un mayor conocimiento de la magnitud del fenómeno, debido a que alguien puede no ser víctima, pero sí perpetrador u observador; e incluso, ejercer varios roles, (c) comparar las ventajas que puede tener la Escala de Ciberacoso con otros instrumentos disponibles. Por último, se recomienda cambiar la escala dicotómica a una escala tipo Likert de 5 puntos: 0 (nunca), 1 (una vez), 2 (dos veces), 3 (tres veces) y 4 (cuatro o más veces); 0 (esto no me ha pasado a mi), 1 (una o dos voces), 2 (pocas semanas), 3 (una vez a la semana), 5 (varias veces a la semana o más), debido a que este tipo de escalas permiten mayor precisión en la evaluación de los constructos.

El estudio presenta varias fortalezas. La muestra permite la representatividad y generalización de los resultados para la población mexicana; los métodos de análisis utilizados son los apropiados para matrices tetracóricas para datos binarios y permiten mayor solidez en los resultados; se incluyen pruebas de invarianza para distintos grupos poblacionales. Además, los análisis son los recomendados por los investigadores que están al frente del campo del estudio del ciberacoso en el mundo.

En conclusión, los análisis de la Escala de Ciberacoso de diez reactivos arrojaron propiedades psicométricas para la población mexicana de 12 a 59 años, que permiten contar con evidencias de validez de constructo $y$ confiabilidad para el estudio de la victimización en el ciberacoso en México. De esta manera, se avanza en el estudio del ciberacoso con una muestra que permite dar cuenta de la estructura factorial en la medición y la generalización de los resultados para el caso mexicano.

\section{Conflicto de Intereses}

Manifestamos que no existe conflicto de intereses por parte de los autores del artículo y que el uso de la base de datos, su interpretación y aplicación en esta investigación no representa una postura oficial del INEGI.

\section{Referencias}

Alonso, C., \& Romero, E. (2020). A longitudinal study of predictors and consequences of cyber bully in spanish adolescents. Behavioral Psychology-Psicologia Conductual, 28(1), 7393.

Álvarez-García, D., Dobarro, A., \& Núñez, J. C. (2015). Validez y fiabilidad del Cuestionario de cibervictimización en estudiantes de Secundaria. Aula Abierta, 43(1), 32-38. https://doi.org/10.1016/j.aula.2014.11.001

Baccarella, C. V., Wagner, T. F., Kietzmann, J. H., \& McCarthy, I. P. (2018). Social media? It's serious! Understanding the dark side of social media. European Management Journal, 36(4), 431-438.

https://doi.org/10.1016/j.emj.2018.07.002

Baier, D., Hong, J. S., Kliem, S., \& Bergmann, M. C. (2019). Consequences of bullying on adolescents' mental health in germany: Comparing face-to-face bullying and cyberbullying. Journal of Child and Family Studies, 28(9), 2347-2357. https://doi.org/10.1007/s10826-018-1181-6

Barlett, C. P. (2019). Cyberbullying, traditional bullying, and aggression: A complicated relationship. In C. P. Barlett (Ed.), Predicting Cyberbullying (pp. 11-16): Academic Press.

Bauman, S., Toomey, R. B., \& Walker, J. L. (2013). Associations among bullying, cyberbullying, and suicide in high school students. Journal of Adolescence, 36(2), 341350.

https://doi.org/10.1016/j.adolescence.2012.12. 001

Bentler, P. M., \& Bonett, D. G. (1980). Significance tests and goodness of fit in the analysis of covariance structures. Psychological Bulletin, 88(3), 588-606. https://doi.org/10.1037/0033-2909.88.3.588

Berne, S., Frisen, A., Schultze-Krumbholz, A., Scheithauer, H., Naruskov, K., Luik, P., Kratzer, C., Erentaite, R., \& Zukauskiene, R. (2013). Cyberbullying assessment instruments: A systematic review. Aggression and Violent Behavior, 18(2), 320-334. https://doi.org/10.1016/j.avb.2012.11.022

Buelga, S., Cava, M. J., \& Musitu, G. (2012). Validation of the adolescent victimization 
through mobile phone and internet scale. Revista Panamericana de Salud Publica-Pan American Journal of Public Health, 32(1), 3642.

https://doi.org/10.1590/s102049892012000700006

Campbell, M., \& Bauman, S. (2018). 1 Cyberbullying: Definition, consequences, prevalence. In M. Campbell \& S. Bauman. Reducing Cyberbullying in Schools, (pp. 316): Academic Press. https://doi.org/10.1016/B978-0-12-8114230.00001-8

Capan, B. E., Bakioglu, F., \& Kirteke, S. (2020). Adaptation of cyber bullying scale to turkish culture: Psychometric properties. Alpha Psychiatry, 21(2), 39-47. https://doi.org/10.5455/apd.57705

Chan, H. C., \& Wong, D. S. W. (2016). Traditional school bullying and cyberbullying perpetration: Examining the psychosocial characteristics of hong kong male and female adolescents. Youth \& Society, 51(1), 3-29. https://doi.org/10.1177/0044118x16658053

Chan, N. N., Ahrumugam, P., Scheithauer, H., Schultze-Krumbholz, A., \& Ooi, P. B. (2020). A hermeneutic phenomenological study of students' and school counsellors' "lived experiences" of cyberbullying and bullying. Computers \& Education, 146, 1-11. https://doi.org/10.1016/j.compedu.2019.103755

Chen, L., Ho, S. S., \& Lwin, M. O. (2017). A meta-analysis of factors predicting cyberbullying perpetration and victimization: From the social cognitive and media effects approach. New Media \& Society, 19(8), 11941213.

https://doi.org/10.1177/1461444816634037

Cheung, G. W., \& Rensvold, R. B. (2002). Evaluating goodness-of-fit indexes for testing measurement invariance. Structural Equation Modeling: A Multidisciplinary Journal, 9(2), 233-255. https://doi.org/10.1207/s15328007sem0902_5

Cross, D., Lester, L., \& Barnes, A. (2015). A longitudinal study of the social and emotional predictors and consequences of cyber and traditional bullying victimization. International Journal of Public Health, 60(2), 207-217. https://doi.org/10.1007/s00038-015-0655-1

Del Rey, R., Casas, J. A., Ortega-Ruiz, R., Schultze-Krumbholz, A., Scheithauer, H., Smith, P., Thompson, F., Barkoukis, V., Tsorbatzoudis, H., Brighi, A., Guarini, A., Pyzalski J., \& Plichta, P. (2015). Structural validation and cross-cultural robustness of the European Cyberbullying Intervention Project Questionnaire. Computers in Human Behavior, 50, 141-147. https://doi.org/10.1016/j.chb.2015.03.065

Flora, D. B., \& Curran, P. J. (2004). An empirical evaluation of alternative methods of estimation for confirmatory factor analysis with ordinal data. Psychological Methods, 9(4), 466-491.

https://doi.org/10.1037/1082-989x.9.4.466

Fluck, J. (2018). Investigating the comparability of two multi-item-scales for cyber bullying measurement. International Journal of Environmental Research and Public Health, 15(11), 9. https://doi.org/10.3390/ijerph15112356

Galvez-Nieto, J. L., Vera-Bachman, D., Cerda, C., \& Diaz, R. (2016). Adolescent victimization through mobile phone and Internet Scale: Psychometric examination in Chilean students. Revista Iberoamericana de Diagnóstico y Evaluación - e Avaliação Psicológica, 41(1), 16-27.

https://www.aidep.org/sites/default/files/articl es/R41/Art2.pdf

Gamez-Guadix, M., Orue, I., Smith, P. K., \& Calvete, E. (2013). Longitudinal and reciprocal relations of cyberbullying with depression, substance use, and problematic internet use among adolescents. Journal of Adolescent Health, 53(4), 446-452. https://doi.org/10.1016/j.jadohealth.2013.03.0 30

Gamez-Guadix, M., Villa-George, F., \& Calvete, E. (2014). Psychometric properties of the Cyberbullying Questionnaire (CBQ) among Mexican adolescents. Violence and Victims, 29(2), 232-247.

https://doi.org/10.1891/0886-6708.Vv-d-1200163r1

Garaigordobil, M. (2015). Psychometric properties of the cyberbullying test, a screening instrument to measure 
cybervictimization, cyberaggression, and cyberobservation. Journal of Interpersonal Violence, 32(23), 3556-3576. https://doi.org/10.1177/0886260515600165

Gini, G., Card, N. A., \& Pozzoli, T. (2018). A meta-analysis of the differential relations of traditional and cyber-victimization with internalizing problems. Aggressive Behavior, 44(2), 185-198. https://doi.org/10.1002/ab.21742

Haid-Stecher, N., Exenberger, S., Unterrainer, C., Bliem, H. R., Sevecke, K., \& Juen, B. (2019). Validation of a german-language version of the multidimensional offline and online peer victimization scale (moopv) in adolescent students. Psychotherapie Psychosomatik Medizinische Psychologie, 70(3/4), 138-144. https://doi.org/10.1055/a-0969-2039

Hinduja, S., \& Patchin, J. W. (2010). Bullying, cyberbullying, and suicide. Archives of Suicide Research, 14(3), 206-221. https://doi.org/10.1080/13811118.2010.494133

Hinduja, S., \& Patchin, J. W. (2018). Connecting adolescent suicide to the severity of bullying and cyberbullying. Journal of School Violence, 18(3), 333-346. https://doi.org/10.1080/15388220.2018.14924 17

Huang, P.-H. (2017). Asymptotics of AIC, BIC, and RMSEA for model selection in structural equation modeling. Psychometrika, 82(2), 407-426. https://doi.org/10.1007/s11336-017-9572-y

Hu, L., \& Bentler, P. M. (1998). Fit indices in covariance structure modeling: Sensitivity to underparameterized model misspecification. Psychological Methods, 3(4), 424-453. https://doi.org/10.1037/1082-989X.3.4.424

Hu, L., \& Bentler, P. M. (1999). Cutoff criteria for fit indexes in covariance structure analysis: Conventional criteria versus new alternatives. Structural Equation Modeling: A Multidisciplinary Journal, 6(1), 1-55. https://doi.org/10.1080/10705519909540118

Instituto Nacional de Estadística y Geografía. (2017a). Módulo sobre Ciberacoso 2017 (MOCIBA) [Diseño Conceptual]. https://www.inegi.org.mx/app/biblioteca/ficha .html?upc $=702825101466$
Instituto Nacional de Estadística y Geografía. (2017b). Módulo sobre Ciberacoso 2017 (MOCIBA) [Nota Técnica]. https://www.inegi.org.mx/contenidos/program as/mociba/2017/doc/mociba2017_nota_tecnic a.pdf

Instituto Nacional de Estadística y Geografía. (2017c). Módulo sobre Ciberacoso 2017 (MOCIBA) [Nota técnica operativa] https://www.inegi.org.mx/contenidos/program as/mociba/2017/doc/mociba2017_nota_tecnic a_operativa.pdf

Instituto Nacional de Estadística y Geografía. (2017d). Módulo sobre Ciberacoso (MOCIBA) 2017 [Base de Datos y descripción de archivos]. https://www.inegi.org.mx/investigacion/cibera coso/2017/default.html\#Microdatos

Kircaburun, K., Demetrovics, Z., \& Tosuntaş, Ş. B. (2019). Analyzing the links between problematic social media use, dark triad traits, and self-esteem. International Journal of Mental Health and Addiction, 17(6), 14961507. https://doi.org/10.1007/s11469-018-9900-1

Kircaburun, K., Kokkinos, C. M., Demetrovics, Z., Kiraly, O., Griffiths, M. D., \& Colak, T. S. (2019). Problematic online behaviors among adolescents and emerging adults: Associations between cyberbullying perpetration, problematic social media use, and psychosocial factors. International Journal of Mental Health and Addiction, 17(4), 891-908. https://doi.org/10.1007/s11469-018-9894-8

Kofoed, J., \& Staksrud, E. (2019). 'We always torment different people, so by definition, we are no bullies': The problem of definitions in cyberbullying research. New Media \& Society, 21(4), 1006-1020. https://doi.org/10.1177/1461444818810026

Kowalski, R. M., \& Limber, S. P. (2013). Psychological, physical, and academic correlates of cyberbullying and traditional bullying. Journal of Adolescent Health, 53(1), S13-S20.

https://doi.org/10.1016/j.jadohealth.2012.09.0 18

Kowalski, R. M., Giumetti, G. W., Schroeder, A. N., \& Lattanner, M. R. (2014). Bullying in the digital age: A critical review and meta- 
analysis of cyberbullying research among youth. Psychological Bulletin, 140(4), 10731137. https://doi.org/10.1037/a0035618

Kwan, I., Dickson, K., Richardson, M., Macdowall, W., Burchett, H., Stansfield, C., Brunton, G., Sutcliffe, K., \& Thomas, J. (2020). Cyberbullying and children and young people's mental health: A systematic map of systematic reviews. Cyberpsychology, Behavior, and Social Networking, 23(2), 7282. https://doi.org/10.1089/cyber.2019.0370

Lee, J., Abell, N., \& Holmes, J. L. (2015). Validation of measures of cyberbullying perpetration and victimization in emerging adulthood. Research on Social Work Practice, 27(4), 456-467. https://doi.org/10.1177/1049731515578535

Ley del Sistema Nacional de Información Estadística y Geografía [LSNIEG], Diario Oficial de la Federación [DOF], 25 de junio de 2018, (México). https://www.snieg.mx/contenidos/espanol/nor matividad/marcojuridico/LSNIEG.pdf

Liang, X., \& Yang, Y. (2014). An evaluation of WLSMV and bayesian methods for confirmatory factor analysis with categorical indicators. International Journal of Quantitative Research in Education, 2(1), 17. https://doi.org/10.1504/ijqre.2014.060972

McDonald, R. P. (1999). Test theory. Psychology Press.

Menesini, E., Nocentini, A., \& Palladino, B. E. (2016). Cyberbullying: Conceptual, theoretical and methodological issues. In T. Völlink, F. Dehue, \& C. McGuckin (Eds.), Cyberbullying: From theory to intervention. (pp. 15-25). Routledge/Taylor \& Francis Group.

Menesini, E., Nocentini, A., Palladino, B. E., Scheithauer, H., Schultze-Krumbholz, A., Frisén, A., \& Blaya, C. (2013). Definitions of cyberbullying. In P. Smith \& G. Steffgen (Eds). Cyberbullying through the New Media: Findings from an International Network (pp. 23-36). Taylor and Francis.

Muthén, B., \& Asparouhov, T. (2014). IRT studies of many groups: The alignment method. Frontiers in Psychology, 5(978). https://doi.org/10.3389/fpsyg.2014.00978
Norma para la difusión y promoción del acceso, conocimiento y uso de la información estadística y geográfica que genera el Instituto Nacional de Estadística y Geografía de 2018 (2018).

https://sc.inegi.org.mx/repositorioNormateca/ On_17Sep18.pdf

Olweus, D. (2012). Cyberbullying: An overrated phenomenon? European Journal of Developmental Psychology, 9(5), 520-538. https://doi.org/10.1080/17405629.2012.682358

Olweus, D., \& Limber, S. P. (2018). Some problems with cyberbullying research. Current Opinion in Psychology, 19, 139-143. https://doi.org/10.1016/j.copsyc.2017.04.012

Ortega-Ruiz, R., Del Rey, R., \& Casas, J. A. (2016). Assessing bullying and cyberbullying: Spanish validation of EBIPQ and ECIPQ. Psicologia Educativa, 22(1), 71-79. https://doi.org/10.1016/j.pse.2016.01.004

Resett, S., \& Caino, P. C. G. (2019). Psychometric properties of a trolling questionnaire in an argentine sample. Revista Argentina De Ciencias del Comportamiento, 11(1), 48-57. https://doi.org/10.32348/1852.4206.v11.n1.20 870

Resett, S., \& Gamez-Guadix, M. (2018). Psychometric properties of cyberbullying questionnaire in an argentinean sample. Universitas Psychologica, 17(5), 1-12. https://doi.org/10.11144/Javeriana.upsy175.ppcc

Rhemtulla, M., Brosseau-Liard, P. É., \& Savalei, V. (2012). When can categorical variables be treated as continuous? A comparison of robust continuous and categorical SEM estimation methods under cuboptimal conditions. Psychological Methods, 17(3), 354-373. https://doi.org/10.1037/a0029315

Schneider, S. K., O’Donnell, L., Stueve, A., \& Coulter, R. W. S. (2012). Cyberbullying, school bullying, and psychological distress: A regional census of high school students. American Journal of Public Health, 102(1), 171-177. https://doi.org/10.2105/ajph.2011.300308

Sittichai, R., \& Smith, P. K. (2018). Bullying and cyberbullying in Thailand: Coping strategies and relation to age, gender, religion and victim status. Journal of New Approaches in 
Educational Research, 7(1), 24-30. https://doi.org/10.7821/naer.2018.1.254

Smith, P. K. (2016). Bullying: definition, types, causes, consequences and intervention. Social and Personality Psychology Compass, 10(9), 519-532. https://doi.org/10.1111/spc3.12266

Smith, P. K., Del Barrio, C., \& Tokunaga, R. S. (2012). Definitions of bullying and cyberbullying: How useful are the terms? In Sh. Bauman, Cross, D. \& J. Walker. Principles of Cyberbullying Research: Definitions, Measures, and Methodology (pp. 26-40). Taylor and Francis.

Smith, P. K., Mahdavi, J., Carvalho, M., Fisher, S., Russell, S., \& Tippett, N. (2008). Cyberbullying: Its nature and impact in secondary school pupils. Journal of Child Psychology and Psychiatry, 49(4), 376-385. https://doi.org/10.1111/j.1469-

7610.2007.01846.x

Solano, A., \& Perugini, M. (2019). Perfiles diferenciales de usuarios de internet, factores de personalidad, rasgos positivos, síntomas psicopatológicos y satisfacción con la vida. Revista Iberoamericana de Diagnóstico y Evaluación - e Avaliação Psicológica, 53(4), 79-90. https://doi.org/10.21865/ridep53.3.06

Términos de libre uso de la Información del Instituto Nacional de Estadística y Geografía de 2019. (2019).

https://www.inegi.org.mx/inegi/terminos.html

Thomas, H. J., Connor, J. P., \& Scott, J. G. (2014). Integrating traditional bullying and cyberbullying: Challenges of definition and measurement in adolescents - a review. Educational Psychology Review, 27(1), 135152. https://doi.org/10.1007/s10648-014-9261-7

Thomas, H. J., Scott, J. G., Coates, J. M., \& Connor, J. P. (2019). Development and validation of the bullying and cyberbullying scale for adolescents: A multi-dimensional measurement model. British Journal of Educational Psychology, 89(1), 75-94. https://doi.org/10.1111/bjep.12223

Twardowska-Staszek, E., Zych, I., \& Ortega-Ruiz, R. (2018). Bullying and cyberbullying in polish elementary and middle schools: Validation of questionnaires and nature of the phenomena. Children and Youth Services Review, 95, 217-225.

https://doi.org/10.1016/j.childyouth.2018.10.045

Van Geel, M., Vedder, P., \& Tanilon, J. (2014). Relationship between peer victimization, cyberbullying, and suicide in children and adolescents a meta-analysis. Jama Pediatrics, 168(5), 435-442.

https://doi.org/10.1001/jamapediatrics.2013.4 143

Vandenberg, R. J., \& Lance, C. E. (2000). A review and synthesis of the measurement invariance literature: Suggestions, practices, and recommendations for organizational research. Organizational Research Methods, 3(1), 4-70. https://doi.org/10.1177/109442810031002

Vranjes, I., Baillien, E., Vandebosch, H., Erreygers, S., \& Witte, H. (2017). When workplace bullying goes online: Construction and validation of the inventory of cyberbullying acts at work (ICA-W). European Journal of Work and Organizational Psychology, 27(1), 28-39. https://doi.org/10.1080/1359432x.2017.1363185

Whittaker, E., \& Kowalski, R. M. (2015). Cyberbullying via social media. Journal of School Violence, 14(1), 11-29. https://doi.org/10.1080/15388220.2014.949377

Williford, A., \& DePaolis, K. J. (2019). Validation of a cyber bullying and victimization measure among elementary school-aged children. Child and Adolescent Social Work Journal, 36(5), 557-570. https://doi.org/10.1007/s10560-018-0583-Z

Wright, M. F., \& Wachs, S. (2019). Adolescents' psychological consequences and cyber victimization: The moderation of schoolbelongingness and ethnicity. International Journal of Environmental Research and Public Health, 16(14), 11. https://doi.org/10.3390/ijerph16142493

Zych, I., Baldry, A. C., Farrington, D. P., \& Llorent, V. J. (2018). Are children involved in cyberbullying low on empathy? A systematic review and meta-analysis of research on empathy versus different cyberbullying roles. Aggression and Violent Behavior, 45, 83-97. https://doi.org/10.1016/j.avb.2018.03.004 
Zych, I., Ortega-Ruiz, R., \& Marín-López, I. (2016). Cyberbullying: A systematic review of research, its prevalence and assessment issues in spanish studies. Psicología

Educativa, 22(1), 5-18.

https://doi.org/10.1016/j.pse.2016.03.002 


\section{Anexo}

\section{Escala de Ciberacoso (ECIBA)}

Instrucción. A continuación, le voy a preguntar si a usted le ha ocurrido alguna de las siguientes situaciones para molestarlo(a) o dañarlo(a).

\section{De junio de 2016 a la fecha, dígame si ¿por teléfono celular o internet alguien...}

1. le envió mensajes ofensivos, con insultos o burlas?

Sí No

2. le hizo llamadas ofensivas, con insultos o burlas?

Sí No

3. publicó información personal, fotos o videos (falsos o verdaderos) de usted para dañarlo(a)?

Sí No

4. lo(a) criticó o se burló de usted en línea por su apariencia o clase social?

5. le hizo insinuaciones o propuestas de tipo sexual?

Sí No

6. se hizo pasar por usted para enviar información falsa, insultar o agredir a

7. lo(a) contactó con nombres falsos para molestarle o dañarle?

8. vigiló sus sitios o cuentas en Internet para causarle molestia o

daño?

lo(a) provocó (molestó o retó) en línea para que reaccione de forma negativa?

10. le envió fotos o videos de contenido sexual para molestarle?
Sí No

Sí No

Sí No

Sí No

Sí No

Sí No 